\title{
Clinico-Pathological Profile and Haematological Abnormalities Associated with Lung Cancer in Bangalore, India
}

\author{
Archana Baburao*, Huliraj Narayanswamy
}

\begin{abstract}
Background: Lung cancer is one of the most common types of cancer causing high morbidity and mortality worldwide. An increasing incidence of lung cancer has been observed in India. Objectives:To evaluate the clinicpathological profile and haematological abnormalities associated with lung cancer in Bangalore, India. Materials and Methods: This prospective study was carried out over a period of 2 years. A total of 96 newly diagnosed and histopathologically confirmed cases of lung cancer were included in the study. Results: Our lung cancer cases had a male to female ratio of 3:1. Distribution of age varied from 40 to 90 years, with a major contribution in the age group between 61 and 80 years $(55.2 \%)$. Smoking was the commonest risk factor found in $69.7 \%$ of patients. The most frequent symptom was cough $(\mathbf{8 6 . 4 \%})$ followed by loss of weight and appetite $(65.6 \%)$ and dyspnea $(64.5 \%)$. The most common radiological presentation was a mass lesion $(55 \%)$. The most common histopathological type was squamous cell carcinoma (47.9\%), followed by adenocarcinoma (28.1\%) and small cell carcinoma (12.5\%). Distant metastasis at presentation was seen in 53.1\% patients. Among the haematological abnormalities, anaemia was seen in $61.4 \%$ of patients, leucocytosis in $36.4 \%$, thrombocytosis in $14.5 \%$ and eosinophilia in $19.7 \%$ of patients. Haematological abnormalities were more commonly seen in non small cell lung cancer. Conclusions: Squamous cell carcinoma was found to be the most common histopathological type and smoking still remains the major risk factor for lung cancer. Haematological abnormalities are frequently observed in lung cancer patients, anaemia being the commonest of all.
\end{abstract}

Keywords: Lung cancer - histopathology - smoking, haematological abnormalities - squamous cell carcinoma

Asian Pac J Cancer Prev, 16 (18), 8235-8238

\section{Introduction}

Lung cancer is the most commonly diagnosed cancer and leading cause of cancer related death worldwide (Alberg et al, 2007). Globally, there are approximately 13 million new cancer cases and 8 million cancer deaths (Ferlay et al, 2010) and the death toll due to lung cancer is projected to rise to 10 million by 2030 with 7 out of 10 deaths in developing world (Thankappan et al, 2007). It is known that lung cancer rates are high in developed countries; nonetheless the rates in developing countries are also rising due to increasing life span and increasing cigarette smoking.

In India, there are approximately 63,000 new lung cancer cases are reported every year (Ganesh et al, 2011). According to the National cancer registry programme 2010 , the rates of lung cancer vary across India. The age adjusted incidence of lung cancer is highest in the north eastern region of India, Aizwal district and lowest in Barshi- a rural registry in western India. The Indian council of medical research(ICMR), after studying lung cancer data over 24 years (1982-2005), has found that while new cases of lung cancer per one lakh male population has increased by around $160 \%$ in Chennai, $100 \%$ in Bangalore and $40 \%$ in Delhi during this period, such cases have fallen by $60 \%$ in Mumbai.

Paraneoplastic syndromes are common in lung cancer, and may be the first manifestation of the disease or its recurrence (Heinemann et al, 2008). Haematological abnormalities are frequently observed in lung cancer patients, anaemia being the commonest of all. Smoking remains the leading cause of lung cancer and both incidence and mortality are directly related to the degree of cigarette use that occurs in a population. However, histopathological cell types may vary with the changes of social and other environmental factors. Population based cancer registries in India give information of site wise distribution of cancer and histological subtypes are not taken into consideration. We undertook this study to analyse the clinico-pathological profile and haematological abnormalities associated with lung cancer at a tertiary care centre, situated in Bangalore.

\section{Materials and Methods}

This prospective study was carried out over a period

Department of Pulmonary Medicine, Kempegowda Institute of Medical Sciences, Bangalore, India *For correspondence: dr_archana82@yahoo.co.in 
of 2 years in the department of Pulmonary medicine, Kempegowda Institute of Medical sciences and research centre, Bangalore, India, which is a tertiary care centre and referral hospital. 96 newly diagnosed and histopathologically confirmed cases of lung cancer were included in the study.

Data regarding demographics, smoking history, clinical presentation, duration of symptoms, radiographic findings, method of diagnosis, histopathological type, clinical stage of the disease and complete heamogram were collected. For confirming the diagnosis of lung cancer, majority of patients were subjected to fibre-optic bronchoscopy and/or CT guided biopsy. Other diagnostic methods used were open biopsy, pleural fluid cytology and supraclavicular lymph node biopsy.

Data analysis was done using SPSS version 20 and results were presented in percentage and simple frequency. Informed written consent was taken from the subjects and ethical clearance was obtained from the ethical committee.

\section{Results}

A total of 96 patients were included in the study, which comprised of $72(75 \%)$ males and $24(25 \%)$ females, with male to female ratio of 3:1. Distribution of age varied from 40 to 90 years. Approximately $74 \%$ of the cases belonged to above 50years of age, with a major contribution in the age group between 61 and 80 years (55.2\%). Smoking was the commonest risk factor found in $69.7 \%$ of patients (Table 1), with $65(90.2 \%)$ male smokers and $2(8.33 \%)$ female smokers and bidi ( $80 \%)$ was the commonest mode of smoking. Majority of male patients were farmers (75\%) and all the female patients were housewives and passive smokers. Most of the female patients also had an exposure to fire wood smoke.

Cough was the commonest presenting complaint present in $83(86.4 \%)$ patients followed by loss of weight and appetite $(65.6 \%)$, dyspnea $(64.5 \%)$, chest pain $(41.6 \%)$ and hemoptysis $(27 \%)$. SVC obstruction was present in $6(6.2 \%)$ patients (Table 2$)$. The duration of complaints varied between 15 days to 6 months with an average of 97 days. Mass lesion (55.2\%) was the commonest radiological feature followed by collapse consolidation (28.1\%), pleural effusion (9.37\%) and combined presentation (7.29\%). Right lung (57.2\%) was the commonest primary site followed by left lung (39.5\%) and both lung $(3.12 \%)$. Various diagnostic modalities, either single or in combination, used for confirmation of lung cancer shown in table 2. Central endobronchial tumours were seen in $56(58.3 \%)$ patients, whereas

Table 1. : Demographic Profile of Lung Cancer Patients $(\mathbf{n}=96)$

\begin{tabular}{llcc}
\hline Age group & & No. Of patients & Percentage \\
\hline \multirow{4}{*}{ Gender } & $40-60$ & 41 & 42.7 \\
& $>60$ & 55 & 57.29 \\
\multirow{3}{*}{ Smokers } & Male & 72 & 75 \\
& Female & 24 & 25 \\
& Male & 65 & 90.27 \\
& Female & 2 & 8.33 \\
\hline
\end{tabular}

Table 2. Clinico-radiological Presentations and Various Diagnostic Modalities Used

\begin{tabular}{lcc}
\hline Variables & No. Of patients & Percent age \\
\hline Symptoms & & \\
Cough & 83 & 86.45 \\
Loss of weight and appetite & 63 & 65.62 \\
Dyspnea & 62 & 64.58 \\
Chest pain & 40 & 41.66 \\
Hemoptysis & 26 & 27.08 \\
Fever & 13 & 13.54 \\
Hoarseness of voice & 12 & 12.5 \\
SVC obstruction & 6 & 6.25 \\
Radiological presentation & & \\
Mass lesion & 53 & 55.2 \\
Collapse-consolidation & 27 & 28.12 \\
Pleural effusion & 9 & 9.37 \\
Combined presentation & 7 & 7.29 \\
Site of primary lung cancer & & \\
Right lung & 55 & 57.29 \\
Left lung & 38 & 39.58 \\
Both lungs & 3 & 3.12 \\
Diagnostic modalities used & & \\
FOB & 66 & 68.75 \\
CT guided biopsy & 22 & 22.91 \\
Supraclavicular lymph node biopsy & 4 & 4.16 \\
Pleural fluid cytology & 2 & 2.08 \\
Open biopsy & 2 & 2.08 \\
\hline
\end{tabular}

Table 3. Histopathological Cell Type of Lung Cancer

\begin{tabular}{lcc}
\hline Histopathological type & No. Of patients & Percent age \\
\hline Squamous cell carcinoma & 46 & 47.91 \\
Adenocarcinoma & 27 & 28.12 \\
Small cell carcinoma & 12 & 12 \\
Large cell carcinoma & 3 & 3.12 \\
Undifferentiated carcinoma & 8 & 8.33 \\
\hline
\end{tabular}

Table 4. Haematological Abnormalities in Lung Cancer

\begin{tabular}{lcc}
\hline Haematological abnormalities & No. Of patients & Percent age \\
\hline Anaemia & 59 & 61.45 \\
Leucocytosis & 35 & 36.45 \\
Thrombocytosis & 14 & 14.58 \\
Eosinophilia & 19 & 19.79
\end{tabular}

peripheral tumours in 30(31.2\%) patients.

The most common histopathological type was squamous cell carcinoma $(47.9 \%)$, followed by adenocarcinoma (28.1\%), small cell carcinoma (12.5\%), undifferentiated carcinoma $(8.33 \%)$ and large cell carcinoma (3.12\%) (Table 3). Squamous cell carcinoma most commonly manifested as central tumour (62.5\%) and adenocarcinoma most commonly manifested as peripheral mass $(73.3 \%)$. Among male patients, squamous cell carcinoma (47.2\%) was the commonest histopathological finding followed by adenocarcinoma (20.8\%) and small cell carcinoma (16.6\%). Whereas in females both squamous cell carcinoma (50\%) and adenocarcinoma $(50 \%)$ had equal rate of occurrence. Nearly $73 \%$ of squamous cell carcinoma and $59 \%$ of adenocarcinoma had smoking history. Distant metastasis at presentation was seen in 51(53.1\%) patients with commonest site as bone $(27.4 \%)$, opposite lung (17.6\%), malignant pleural 
Clinico-Pathological Profile and Haematological Abnormalities Associated with Lung Cancer in Bangalore, India

effusion $(15.6 \%)$, liver $(11.7 \%)$, adrenals $(7.84 \%)$ and multiple sites $(19.6 \%)$. Direct extension with regional lymph nodes was seen in $14(14.5 \%)$ patients. More over $12(12.5 \%)$ patients were in too advanced condition.

Among the haematological abnormalities, anaemia was seen in $59(61.4 \%)$ patients, leucocytosis in 35 $(36.4 \%)$, thrombocytosis in $14(14.5 \%)$ and eosinophilia in $19(19.7 \%$ ) patients (Table 4). Haematological abnormalities were more commonly seen in non small cell lung cancer with thrombocytosis occurring solely in non small cell lung cancer.

\section{Discussion}

Primary lung cancer in India was rare during the early 20th century. But now with increased life span and increasing prevalence of smoking, lung cancer has reached an epidemic proportion in India (Behera and Balamugesh, 2004). It has become a major health problem. The Indian council of medical research (ICMR) has found that the new cases of lung cancer per one lakh male population has increased by $100 \%$ in Bangalore over the past 24 years. Our study included a total of 96 patients with male to female ration of $3: 1$ which is similar to to study (Bahader and Jazieh 2008). This is contrary to other Indian studies that reported male to female of 8.2:1 (Rawat et al, 2009). However in developed countries the incidence of lung cancer among women is on rise due to increase in cigarette smoking among women. The highest incidence of lung cancer was found in sixth and seventh decade of life which was similar to most of the Indian studies with the average age at the time of diagnosis of 61.8 years. We did not find any particular relationship between the age and histological cell type.

Smoking was the commonest cause of lung cancer (Vineis et al 2004). Smoking was prevalent among $90.27 \%$ of male patients and $8.33 \%$ female patients only. Majority of male patients were farmers $(75 \%)$ and all the female patients were housewives with most of them having been exposed to fire wood smoke. Further in our study, there was a high incidence of lung cancer among females even though only $8.33 \%$ female patients had a smoking history. These indicate that factors other than active smoking also play an important role in causing lung cancer like use of fire wood for cooking, passive smoking among these females and use of pesticides. Smoking was associated with all types of lung cancer in our study. The connection between smoking and lung cancer was most evident among squamous cell carcinoma, small cell carcinoma and large cell and less evident with adenocarcinoma. This result is similar to that reported in another study (Khuder 2001).

Clinical presentation of the patients showed that cough was predominant symptom followed by breathlessness, loss of weight and appetite, chest pain and hemoptysis. This is similar to reports published from different parts of India (Behera et al 2004, Rawat et al 2009). The most common radiological presentation seen in our study was mass lesion followed by collapse consolidation, pleural effusion and combined presentation similar to reports published in Indian literature (Rawat et al 2009, Mandal et al 2013) with predominantly involving right lung at presentation. Bronchoscopy was the commonest diagnostic tool used in patients with suspected endobronchial tumour which was similar to other study. Peripherally situated mass lesions which were beyond the bronchoscopic reach were diagnosed with CT guided biopsies.

The pattern of lung cancer is changing in the developed countries. The rate of lung cancer among females is increasing and adenocarcinoma has over taken squamous cell carcinoma as the most common histological cell type. However the pattern seen in our study was different. Squamous cell carcinoma was still the commonest cell type in both the sexes followed by adenocarcinoma and small cell carcinoma which is similar to reports from other Indian studies (Behera et al 2004, Rawat et al 2009, Mandal et al 2013). This may be due to the fact that active smoking is less prevalent among Indian women as opposed to west. Squamous cell carcinoma was more prevalent among smokers and manifested more commonly as central tumour and adenocarcinoma as peripheral mass lesion which was in agreement with other studies (Rawat et al 2009). Distant metastasis at presentation was seen in $51(53.1 \%)$ patients in our study with commonest site as bone, opposite lung, malignant pleural effusion, liver, adrenals and multiple sites which is similar to other Indian studies(Khan et al 2006, Malik et al 2014). Most of the patients in our study presented in more advanced stage. This delay in presentation may be due lack of knowledge about the disease, ignorance, delay in seeking medical help, misdiagnosis and poor referral to speciality centres

Haematological abnormalities were more commonly seen in our patients. The incidence of haematological abnormalities was similar to another study. (Ferrigno et al 2003). The cause of anaemia in lung cancer is multi factorial. Bleeding, hemolysis, bone marrow infiltration and nutritional deficiencies may all contribute to the development of anaemia. Inflammatory cytokines such as TNF-a, IL-1, IL-6 inhibits erythropoiesis resulting in anaemia. Anaemia in the context of a malignancy predicts a poor outcome ((Langer et al, 2002). Leucocytosis is thought to be due to overproduction of granulocyte colony stimulating factor (Kasuga et al, 2001). Tumour related leucocytosis is associated with poor prognosis compared to patients without leucocytosis. Thrombocytosis is also relatively common in lung cancer patients with a prevalence of 16-32\% (Aoe et al, 2004) which was similar to our study. Thrombocytosis in lung cancer is considered to be a part of systemic inflammatory reaction and is an independent predictor of poor prognosis in these patients (Aoe et al, 2004). IL-5 has been implicated as a causative factor for eosinophilia in lung cancer. Especially non small cell lung cancer is associated with excessive eosinophilia (Pandit et al, 2007).

To conclude higher incidence of lung cancer was found in sixth and seventh decade of life with locally and distantly advanced stages. Gender gap is becoming narrow, as one third of diagnosed patients are women in our study. Smoking was the commonest cause of lung cancer. Squamous cell carcinoma was the commonest histological type followed by adenocarcinoma and small cell carcinoma. Haematological abnormalities 


\section{References}

Alberg AJ, Ford JG, Samet JM (2007). Epidemiology of lung ACCP evidence-based clinical practice guidelines cancer. Chest, 132, 29-55.

Aoe K, Hiraki A, Ueoka H, et al (2004). Thrombocytosis as a useful prognostic indicator in patients with lung cancer. Respiration, 71, 170-73.

Bahader Y, Jazieh A (2008). Epidemiology of lung cancer. Ann Thorac Med, 3, 365-67.

Behera D, Balamugesh T (2004). Lung cancer in India. Indian $J$ Chest Dis Allied Sci, 46, 269-81.

Ferlay J, Shin H R, Bray F, et al (2010). GLOBOCAN 2008. Cancer Incidence and mortality Worldwide: IARC Cancer Base No.10. International Agency for Research on cancer, Lyon, France.

Ferrigno D, Buccheri G (2003). Hematologic counts and clinical correlates in 1201 newly diagnosed lung cancer patients. Monaldi Arch Chest Dis, 59, 193-98.

Ganesh B, Sushma S, Monika S, et al (2011). A case control study of risk factors for lung cancer in Mumbai, India. Asian Pac J Cancer Prev, 12, 357-62.

Kasuga I, Makino S, Kiyokawa H, et al (2001). Tumour related leucocytosis is linked with poor prognosis in patients with lung carcinoma. Cancer, 92, 2399-405.

Khan NA, Afroz F, Lone MM, et al (2006). Profile of lung cancer in Kashmir, India-A five year study. Indian J Chest Dia Allied Sci, 48, 187-90.

Khuder SA (2001). Effect of cigarette smoking on major histological types of lung cancer: a meta analysis. Lung cancer, 31, 139-48.

Langer CJ, Choy H, Glaspy JA, et al (2002). Standards of care for anemia management in oncology: focus on lung carcinoma. Cancer, 95, 613-23.

Malik PS, Sharma MC, Mohanti BK, et al (2013). Clinocopathological profile of lung cancer at AIIMS: a changing paradigm in India. Asian Pac J Cancer Prev, 14, 489-94.

Mandal SK, Singh TT, SharmaTD, et al (2013). Clinicopathology of lung cancer in a regional cancer center in northeastern India. Asian Pac J Cancer Prev, 14, 7277-81.

Pandit R, Scholnik A, Wulfekuhler L, et al (2007). Non- smallcell lung cancer associated with excessive eosinophilia and secretion of interleukin-5 as a paraneoplastic syndrome. Am J Hematol, 82, 234-37.

Rawat J, Sindhwani G, Gaur D, et al (2009). Clinico-pathological profile of lung cancer. Lung India, 26, 74-76.

Stafanie Heinemann, Peter Zabel, Hams-Peter Hauber (2008). Paraneoplastic syndromes in lung cancer. Cancer therapy, 6, 687-98.

Thankappan KR, Thresia CU (2007). Tobacco use and social status in Kerala. Indian J Med Res, 126, 300-8.

Vineis P, Alavanja M, Buffler P, et al (2004). Tobacco and cancer: recent epidemiological evidence. J Natl Cancer Inst, 96, 99-106. 